\title{
Long-Term Benefits of Tailored Exercise in Severe Sarcoidosis: A Case Report
}

\author{
Alba M. Herrera-Olivares ${ }^{1}\left(\mathbb{D}\right.$, Juan M. García-Manso ${ }^{2}$, Irene Rodríguez-Gómez ${ }^{3,4}$ (), \\ Ignacio Ara ${ }^{3,4} \mathbb{D}$, Alejandro Lucia ${ }^{1,4,5, *}$ and Alfredo Santalla $5,6 \mathbb{D}$ \\ 1 Faculty of Sport Sciences, Universidad Europea de Madrid, 18670 Madrid, Spain; amheroli@gmail.com \\ 2 Hospital San Juan de Dios, Santa Cruz de Tenerife, 38009 Canary Islands, Spain; jgarciamanso@gmail.com \\ 3 GENUD Toledo Research Group, Universidad de Castilla-La Mancha, 45004 Toledo, Spain; \\ Irene.Rodriguez@uclm.es (I.R.-G.); Ignacio.ara@uclm.es (I.A.) \\ 4 CIBER of Frailty and Healthy Aging (CIBERFES), 28029 Madrid, Spain \\ 5 Instituto de Investigación Hospital 12 de Octubre (imas12), 28041 Madrid, Spain; asanher@upo.es \\ 6 Faculty of Sport Sciences, Universidad Pablo de Olavide, 41013 Seville, Spain \\ * Correspondence: alejandro.lucia@universidadeuropea.es; Tel.: +34-6613-93101; Fax: +34-9161-68265
}

Received: 25 October 2020; Accepted: 12 December 2020; Published: 18 December 2020

\begin{abstract}
Background: We studied the effects of a supervised, structured exercise program in a severe sarcoidosis patient. Methods: After being clinically stable for two years, a 52-year-old woman (stage IV, American Thoracic Society) who originally had irreversible lung fibrosis, pulmonary arterial hypertension (PAH), mild mitral insufficiency, and atrial dilatation, and was candidate for lung transplant, performed a combined high-intensity interval, high load resistance, and inspiratory muscle training for 4.5 years, and was tested (cardiopulmonary exercise testing and dual X-ray absorptiometry) every six months. Results: Cardiorespiratory fitness (CRF) and maximal pulmonary ventilation increased by $44 \%$ and $60 \%$, respectively. Ventilatory efficiency also improved (decrease in the ventilatory equivalent for oxygen by $32 \%$ and $14 \%$ at the ventilatory threshold and respiratory compensation point, respectively). She improved New York Heart Association (NYHA) class (from III to II), and cardiac alterations as well as PAH reversed so that she was not in need of lung transplantation anymore. Likewise, she suffered no more episodes of hemoptysis. Bone health was overall maintained despite the post-menopausal status and the corticoid treatment. Conclusions: A long-term combined exercise intervention safely contributed-at least partly-to improve CRF and NYHA class in a patient with severe sarcoidosis, suggesting a potential coadjuvant effect to attenuate clinical manifestations.
\end{abstract}

Keywords: cardiopulmonary exercise test; pulmonary fibrosis; high-intensity interval training; inspiratory muscle training

\section{Introduction}

Sarcoidosis is an autoimmune disease of unknown etiology (incidence of 2.17-519/ 100,000 people/year [1]), characterized by the presence of noncaseating granulomas in the affected organs [2]. The condition is more common in women than men, with a peak age of onset of 30-55 years. There is high individual variability in the clinical manifestations, and many patients are asymptomatic or will have remission of symptoms within two years [3]. Sarcoidosis can affect several organs, but it preferentially affects lymph nodes and the lung where it can cause different levels of damage ranging from asymptomatic involvement to severe manifestations, including pulmonary fibrosis [4]. Pulmonary vessels might also be affected, which increase the risk of a life-threatening complication-severe pulmonary arterial hypertension (PAH) [5]. 
Fatigue is the most commonly reported symptom of sarcoidosis [3], which together with dyspnea, persistent cough, and physical limitations, can impair the quality of life (QoL) of symptomatic patients and lead to anxiety, depression, and social isolation [6]. Patients commonly have low levels of physical activity (PA) $[7,8]$ and functional capacity $[7,9]$ and, in fact, international experts recommend the implementation of exercise for treating symptomatic patients [10]. However, there is still a lack of evidence as to which training program is best (type of exercise, intensity, frequency, or duration) for this patient population [10]. In general, physical exercise interventions in patients with sarcoidosis have combined aerobic and strength exercises (as well as breathing exercises) for a maximum duration of 13 weeks [11-15]. The typical aerobic component consists of moderate-intensity continuous training (MICT, e.g., 20-30 min of walking/cycling at $\leq 70 \%$ of maximal aerobic capacity). In this regard, a type of exercise training that is gaining popularity in Western societies is high-intensity interval training (HIIT), because it is thought to stimulate aerobic fitness and muscle molecular adaptations that are comparable - if not superior - to those elicited by MICT despite a lower time commitment [16]. However, no study has yet assessed the long-term effects (or "adaptations") induced by HIIT in symptomatic patients with sarcoidosis, in part because the potential exacerbation of the feeling of fatigue with intense exertion is a concern in this population [12]. Preliminary evidence has, however, indicated that a single HIIT session does not affect fatigue differently from a single MICT session in patients with sarcoidosis, supporting the need for further research on the long-term effects of HIIT for these patients [17].

Approximately $10 \%$ of patients with sarcoidosis develop end-stage fibrotic lung disease with high indication for lung transplantation [18]. Prior to this, patients may experience long-term and progressive muscle weakness [19], also affecting the inspiratory musculature [20]. Accordingly, it would be of clinical value to provide preliminary (or "proof-of-concept") evidence on the potential benefits of programmed exercise performed over the years in those patients with a worse prognosis. Here we present the case of a patient with systemic sarcoidosis and associated severe lung affectation who underwent a supervised combined (HIIT + high load resistance + inspiratory muscle training (IMT)) program over a period of 4.5 years.

\section{Experimental Section (Case Report)}

The patient is a post-menopausal woman aged 52 years at the start of the study (height $162 \mathrm{~cm}$; weight, $54 \mathrm{~kg}$; body mass index, $20.6 \mathrm{~kg} \cdot \mathrm{m}^{-2}$ ), diagnosed in 2007 with the most severe presentation of systemic sarcoidosis according to the American Thoracic Society criteria-stage IV-indicating irreversible scarring in the lungs (pulmonary fibrosis) [21], in the Pulmonary Unit of the Hospital Universitario de Gran Canaria Doctor Negrín (Canary Islands, Spain).

Beyond pulmonary involvement (which also included bilateral peribronchial and mediastinal calcified lymphadenopathy and iatrogenic pneumothorax), she had ocular, dermal, and articular symptoms (Figure 1). She has been treated with corticosteroids since diagnosis and has a history of recurrent exacerbations and hemoptysis episodes of infectious origin. In 2013, she had an episode of severe acute pulmonary thromboembolism and was diagnosed with moderate $\mathrm{PAH}$, diastolic atrial dilation, and mild mitral insufficiency; she was classified as New York Heart Association (NYHA) class IV. For this reason, she was also treated with anticoagulants and was considered as a candidate for eventual lung transplantation. Two years later, despite being clinically stable and having improved her status such as to be classified as NYHA class III, the patient reported a marked limitation in physical activities. She also reported that she needed to rest every $\sim 100 \mathrm{~m}$ when walking in order to recover from the associated dyspnea, with recurrent episodes of hemoptysis also triggered by exertion.

She practiced athletics from the age of 17 to 20 years and adopted an essentially sedentary lifestyle since age 23 until starting our intervention-except for two 12-week respiratory rehabilitation programs performed 4 and 3 years, respectively, before the start of our exercise training intervention. 


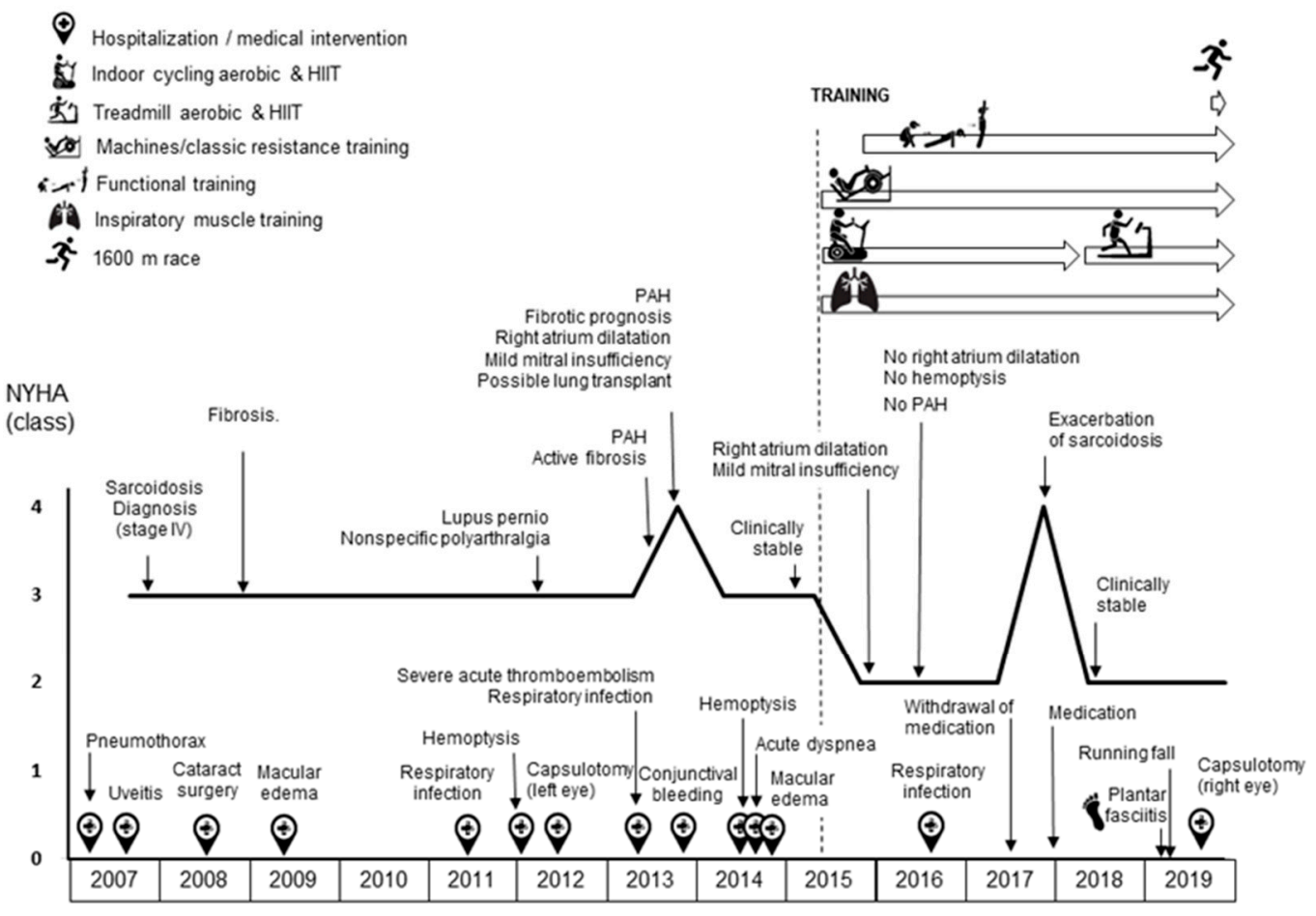

Figure 1. Graphical Abstract. Clinical and functional evolution of the patient since diagnosis of sarcoidosis. Abbreviations: HIIT, high-intensity interval training; NYHA, New York Heart Association; $\mathrm{PAH}$, pulmonary arterial hypertension.

\subsection{Assessments}

All assessments were performed every 6 months (June and December) at the Exercise Physiology Laboratory at Hospital 12 de Octubre (Madrid, Spain), which she first visited in June 2015. After we studied her medical history in depth (with one of us (A.L.) being a physician with solid expertise in the implementation of exercise interventions, including in-hospital programs, for patients with numerous disease conditions such as PAH [22]), she underwent a battery of physical capacity assessments (as described below) every six months. She gave her written consent to participate in the study and to have her data published. The study was conducted in accordance with the Declaration of Helsinki, and the protocol was approved by the Ethics Committee of the aforementioned institution (approval number: 13/377).

First, we measured maximum inspiratory pressure (MIP) (RPM, Micro Medical Inc., Chatham, Kent, UK). Due to the history of recurrent hemoptysis (some during spirometry) and the fact that performing forced expiration is known to increase pulmonary capillary pressure [23], we refrained from performing spirometry or maximum expiratory pressure assessments.

Thereafter, the patient performed a cycle ergometer (Ergometrics 900, Ergoline, Barcelona, Spain) exercise test until volitional exhaustion, during which blood pressure (BP), heart rate (HR), 12-lead ECG, peripheral oxygen saturation $\left(\mathrm{SpO}_{2}\right)$ (TRUSAT 3500, General Electric Oy; Helsinki, Finland) and gas exchange data (CPX Ultima, Medical Graphics Corporation, St Paul, MN, USA) were continuously monitored. We followed a published protocol [24] with slight modifications. Thus, after a 2-min resting period on the ergometer, the patient pedaled for $2 \mathrm{~min}$ at a cadence of 60-65 rpm without resistance ( 0 watt). The workload was then increased by 1 watt every $6 \mathrm{~s}$ (averaging $10 \mathrm{watts} / \mathrm{min}$ ) until exhaustion or until $\mathrm{SpO}_{2}$ decreased below $90 \%$. Other criteria for test termination were chest pain, ECG signs of ischemia, severe dyspnea disproportionate to the effort, tachyarrhythmias, or a drop in $\mathrm{BP}$. The protocol also included a recovery phase, during which the patient pedaled for one minute at 10 watts and one minute without resistance, before finally resting for one minute on the ergometer 
to prevent post-exercise desaturation. Gas-exchange parameters (10-s averages) where collected for the determination of the peak value of oxygen uptake $\left(\mathrm{VO}_{2}\right.$ peak) reached during the tests, as well as the ventilatory threshold (VT) and respiratory compensation threshold (RCP, also termed "second ventilatory threshold"). The VT corresponded to the workload at which the ventilatory equivalent for oxygen (ventilation $\left(\mathrm{VE} \cdot \mathrm{VO}_{2}{ }^{-1}\right)$ starts to increase with no concomitant increase in the ventilatory equivalent for carbon dioxide $\left(\mathrm{VE} \cdot \mathrm{VCO}_{2}{ }^{-1}\right)$ and with departure from linearity of VE. The RCP was determined as the workload at which both $\mathrm{VE} \cdot \mathrm{VO}_{2}{ }^{-1}$ and $\mathrm{VE} \cdot \mathrm{VCO}_{2}{ }^{-1}$ increase together with a decrease in end-tidal pressure of carbon dioxide [25].

In the first visit only, the patient performed a circuit strength training session using basic machines (chest press, leg press, lateral pull down, and abdominals), during which we monitored $\mathrm{SpO}_{2}$ to analyze her $\mathrm{SpO}_{2}$ response (and eventual drops of this variable) during and after each set of exercises.

After the first year of training, we incorporated body composition analysis (dual X-ray absorptiometry; Hologic Discovery QDR Series, Software Physician's Viewer, APEX System Software Version 3.1.2. Bedford, MA, USA) to the battery of assessments. Body mass composition was calculated from a full body study scan, and a specific analysis of bone density in the spine $\left(\mathrm{L}_{1}-\mathrm{L}_{4}\right)$ and femoral neck was performed as described [26].

\subsection{Exercise Training Intervention}

The patient underwent a combined (HIIT + high load resistance + IMT) program for a total of 4.5 years in her local fitness center under the supervision of a fitness specialist. The patient generally trained five to six days/week. This included two sessions of strength training using machines, two sessions of strength training using "functional exercises", and two HIIT sessions. When she performed two sessions per day, this consistently included a HIIT and a functional training session, respectively.

The HIIT program is detailed in Table 1. Heart rate and $\mathrm{SpO}_{2}$ (TRUSAT 3500, General Electric Oy; Helsinki, Finland), and rating of perceived exertion (RPE, on a 0 to 10 scale) were recorded in each session. During the first three years all the sessions were performed on a cycle-ergometer (S-UBx, Star Trac, USA). To prevent eventual abrupt decreases in $\mathrm{SpO}_{2}$ after exertion, each session ended with a cool-down phase ( 6 min of pedaling at 15 watts with a cadence of $60 \mathrm{rpm}$, followed by 2 min during which resistance was gradually reduced to 0 ). In the last phase (1.5 years) of the program, the HIIT sessions were performed on a treadmill (S-TRc, Star Trac, CA). The transition from HIIT cycle-ergometer to HIIT treadmill sessions served two purposes: (i) to apply a more functional exercise modality with a greater transference to daily life activities; and (ii) to increase the impact load associated with exercise in order to prevent—or at least attenuate- the loss of bone mineral density that is known to be associated with both post-menopause and corticosteroid treatment $[27,28]$.

In the last month of the follow-up, and with the goal of further motivating the patient, she completed a one-mile running race at an average speed of $6.4 \mathrm{~km} / \mathrm{h}$ (range 6-7) with $\mathrm{SpO}_{2}$ consistently $>90 \%$. Of note, this race speed was similar to the one corresponding to the active rest periods between intervals in the treadmill HIIT sessions (Table 1).

Strength training program is detailed in Table 2. It included the same circuit on machines (chest press, leg press, lateral pull down, and abdominal) as on the first visit and was performed twice weekly. The load was progressively increased to 4 series of 7 repetitions in the sixth month. Thereafter, chest press was replaced by a modified chest fly exercise, performed with the trunk titled $\sim 10^{\circ}$ forward and with grip over the shoulder (Video S1), and the lateral pull down was changed to a behind-the-neck lateral pull down executed with the trunk tilted $10^{\circ}$ forward (Video S2). These variations were intended to expand the rib cage against the patient's manifest dorsal hyperkyphosis. 
Table 1. High-intensity interval training program.

\begin{tabular}{|c|c|c|c|c|c|c|c|c|c|c|c|c|}
\hline \multirow[b]{2}{*}{ Period } & \multirow[b]{2}{*}{$\begin{array}{l}\text { Days/ } \\
\text { Week }\end{array}$} & \multirow[b]{2}{*}{$\begin{array}{l}\text { Exercise } \\
\text { Mode }\end{array}$} & \multirow{2}{*}{$\begin{array}{c}\text { Total } \\
\text { Session } \\
\text { Duration } \\
\text { (Min) }\end{array}$} & \multicolumn{4}{|c|}{ Between Intervals } & \multicolumn{5}{|c|}{ Intervals/Sprints } \\
\hline & & & & $\begin{array}{c}\text { Average } \\
\text { Internal Load }\end{array}$ & $\begin{array}{l}\text { Average HR } \\
\text { (Beats-Min }^{-1} \text { ) }\end{array}$ & $\begin{array}{l}\text { Average } \\
\% \mathrm{HR} \max \end{array}$ & $\begin{array}{c}\text { Average } \\
\mathrm{SpO}_{2}(\%)\end{array}$ & Number $\times$ Duration/Recovery & $\begin{array}{c}\text { RPE } \\
(0-10)\end{array}$ & $\begin{array}{c}\text { Average HR } \\
\text { (Beats:Min }{ }^{-1} \text { ) }\end{array}$ & $\begin{array}{c}\text { HR } \\
\text { (\%HRmax) }\end{array}$ & $\begin{array}{c}\text { Average } \\
\mathrm{SPO}_{2} \mathrm{MIN} \\
(\%)\end{array}$ \\
\hline July-September 2015 & 4 & Cycling & 30 & $50 \%$ (VT-RCP) & 125 & 74 & 96 & $10-15 \times 8 \mathrm{~s} / 2-3 \mathrm{~min}$ & 9 & 130 & 77 & 93 \\
\hline October-December 2015 & $3-4$ & Cycling & $40-60$ & $50 \%$ (VT-RCP) & 132 & 79 & 94 & $14-30 \times 8 \mathrm{~s} / 2-3 \mathrm{~min}$ & 8 & 135 & 80 & 93 \\
\hline January-March 2016 & $3-4$ & Cycling & 60 & $50 \%$ (VT-RCP) & 135 & 81 & 95 & $30 \times 5-10 \mathrm{~s} / 1 \mathrm{~min}$ & 7 & 141 & 84 & 91 \\
\hline April-June 2016 & 3 & Cycling & 60 & $50 \%$ (VT-RCP) & 125 & 75 & 94 & $30 \times 5 \mathrm{~s} / 1 \mathrm{~min}$ & 7 & 135 & 81 & 91 \\
\hline July-September 2016 & 3 & Cycling & $60-45$ & $50 \%$ (VT-RCP) & 125 & 75 & 95 & $30-23 \times 10 \mathrm{~s} / 2 \mathrm{~min}$ & 7 & 135 & 81 & 90 \\
\hline October-November 2016 & $3-4$ & Cycling & 45 & $50 \%$ (VT-RCP) & 125 & 75 & 94 & $23 \times 10 \mathrm{~s} / 2 \mathrm{~min}$ & 7 & 135 & 81 & 90 \\
\hline December 2016 & \multicolumn{12}{|c|}{ Decrease in medication (corticosteroids) } \\
\hline January-March 2017 & 3 & Cycling & $45-60$ & $50 \%$ (VT-RCP) & 135 & & 94 & $23-30 \times 10 \mathrm{~s} / 2 \mathrm{~min}$ & 8 & 145 & 87 & 90 \\
\hline April 2017 & 2 & Cycling & 60 & $50 \%$ (VT-RCP) & 135 & 81 & 94 & $30 \times 10 \mathrm{~s} / 2 \mathrm{~min}$ & 8 & 145 & 87 & 89 \\
\hline May-June 2017 & \multicolumn{12}{|c|}{ Progressive withdrawal of medication } \\
\hline July-September 2017 & $2-3$ & Cycling & $30-60$ & $50 \%$ (VT-RCP) & 130 & 78 & 94 & $15-30 \times 10 \mathrm{~s} / 2 \mathrm{~min}$ & 7 & 145 & 87 & 90 \\
\hline October-November 2017 & 3 & Cycling & $45-60$ & $50 \%$ (VT-RCP) & 135 & 81 & 94 & $23-30 \times 10 \mathrm{~s} / 2 \mathrm{~min}$ & 7 & 145 & 87 & 90 \\
\hline December 2017 & \multicolumn{12}{|c|}{ Exacerbation of sarcoidosis } \\
\hline January-March 2018 & 2 & Cycling & 60 & $50 \%(\mathrm{VT}-\mathrm{RCP})$ & 135 & 82 & 94 & $30 \times 10 \mathrm{~s} / 2 \mathrm{~min}$ & 6 & 143 & 87 & 89 \\
\hline $\begin{array}{l}\text { April-June } 2018 \\
\text { A }\end{array}$ & 3 & Running & 30 & $7 \mathrm{~km} \cdot \mathrm{h}^{-1}$ & 135 & 82 & 90 & $12 \times 15 \mathrm{~s}\left(15 \mathrm{~km} \cdot \mathrm{h}^{-1}\right) / 2.5 \mathrm{~min}$ & 9 & 140 & 85 & 89 \\
\hline July-September 2018 & $2-3$ & Running & $40-60$ & $7 \mathrm{~km} \cdot \mathrm{h}^{-1}$ & 140 & 85 & 92 & $16-24 \times 15 \mathrm{~s}\left(15 \mathrm{~km} \cdot \mathrm{h}^{-1}\right) / 2.5 \mathrm{~min}$ & 9 & 155 & 94 & 91 \\
\hline October-December 2018 & 2 & Running & $40-60$ & $7 \mathrm{~km} \cdot \mathrm{h}^{-1}$ & 142 & 86 & 93 & $16-24 \times 15 \mathrm{~s}\left(15-18 \mathrm{~km} \cdot \mathrm{h}^{-1}\right) / 2.5 \mathrm{~min}$ & 9 & 165 & 100 & 89 \\
\hline January-March 2019 & \multicolumn{12}{|c|}{ Plantar fasciitis } \\
\hline April-June 2019 & 2 & Cycling & $30-45$ & $50 \%$ (VT-RCP) & 135 & 82 & 93 & $10-23 \times 8 \mathrm{~s} / 2-3 \mathrm{~min}$ & 5 & 145 & 88 & 89 \\
\hline July-September 2019 & 2 & Running & 45 & $7 \mathrm{~km} \cdot \mathrm{h}^{-1}$ & 135 & 82 & 94 & $23 \times 10 \mathrm{~s}\left(15 \mathrm{~km} \cdot \mathrm{h}^{-1}\right) / 2 \mathrm{~min} *$ & 9 & 155 & 95 & 85 \\
\hline October-December 2019 & 2 & Running & $50-45$ & $7 \mathrm{~km} \cdot \mathrm{h}^{-1}$ & 135 & 82 & 94 & $25-23 \times 10 \mathrm{~s}\left(15 \mathrm{~km} \cdot \mathrm{h}^{-1}\right) / 2 \mathrm{~min} * *$ & 9 & 155 & 95 & 85 \\
\hline
\end{tabular}

Abbreviations: HR, heart rate; HRmax, age-predicted maximum heart rate (220 minus age, in years); HRpeak, peak heart rate achieved after sprint; RPE, rating of perceived exertion in

Borg scale (0-10); $\mathrm{SpO}_{2}$, peripheral oxygen saturation; $\mathrm{SpO}_{2} \mathrm{~min}$, minimum value of $\mathrm{SpO}_{2}$ after a sprint; $50 \%$ (VT-RCP), internal (i.e., heart rate-based) work load equidistant between the

ventilatory threshold and the respiratory compensation point. Symbols: * $3 \mathrm{~kg}$ weighted vest; ** $4 \mathrm{~kg}$ weighted vest. 
Table 2. Resistance training program.

\begin{tabular}{|c|c|c|c|c|c|c|c|c|c|c|c|c|}
\hline \multirow[b]{2}{*}{ Period } & \multirow[b]{2}{*}{$\begin{array}{l}\text { Days/ } \\
\text { Week }\end{array}$} & \multicolumn{6}{|c|}{ Classic Resistance Training } & \multirow[b]{2}{*}{$\begin{array}{l}\text { Days/ } \\
\text { Week }\end{array}$} & \multicolumn{4}{|c|}{ Functional Training } \\
\hline & & $\begin{array}{l}\text { Modified Chest } \\
\text { Fly Machine* }\end{array}$ & Leg Press & $\begin{array}{c}\text { Modified } \\
\text { Behind the } \\
\text { Neck Lat } \\
\text { Pulldown ** }\end{array}$ & $\underset{* * * *}{\text { Planks }}$ & $\begin{array}{c}\text { Russian Belt } \\
\text { Squat }\end{array}$ & $\begin{array}{l}\text { Clean \& } \\
\text { Jerk/Split }\end{array}$ & & $\begin{array}{l}\text { Step-Full } \\
\text { Squat }\end{array}$ & Lunges & Squat Jumps & Burpees \\
\hline July-September 2015 & 2 & $\begin{array}{c}3 \times 5-10 \mathrm{~kg} \\
(8-92 \%)\end{array}$ & $\begin{array}{c}3 \times 6-10 / 30 \mathrm{~kg} \\
(6-94 \%)\end{array}$ & $\begin{array}{c}3 \times 5-15 \mathrm{~kg} \\
(7-88 \%)\end{array}$ & $\begin{array}{l}2 / 4 \times 6 \\
(8-94 \%)\end{array}$ & & & & & & & \\
\hline October-December 2015 & 2 & $\begin{array}{c}4 \times 7-10 \mathrm{~kg} \\
(8-91 \%)\end{array}$ & $\begin{array}{c}4 \times 7-30 / 40 \mathrm{~kg} \\
(6-94 \%)\end{array}$ & $\begin{array}{c}4 \times 7-15 \mathrm{~kg} \\
(7-88 \%)\end{array}$ & $\begin{array}{c}4 \times 7 \\
(7-92 \%)\end{array}$ & & & & & & & \\
\hline January-March 2016 & 2 & $\begin{array}{c}4 \times 8-15 \mathrm{~kg} \\
(9-91 \%)\end{array}$ & $\begin{array}{c}4 \times 8-50 \mathrm{~kg} \\
(6-95 \%)\end{array}$ & $\begin{array}{c}4 \times 8-25 \mathrm{~kg} \\
(8-88 \%)\end{array}$ & $\begin{array}{c}4 \times 8 \\
(7-94 \%)\end{array}$ & & & 2 & $3 \times 15(8-90 \%)$ & $3 \times 15(5-93 \%)$ & $3 \times 1 / 12(7-90 \%)$ & \\
\hline April-June 2016 & 2 & $\begin{array}{c}4 \times 8-15 \mathrm{~kg} \\
(9-92 \%)\end{array}$ & $\begin{array}{c}4 \times 8-50 \mathrm{~kg} \\
(6-95 \%)\end{array}$ & $\begin{array}{c}4 \times 8-25 \mathrm{~kg} \\
(8-88 \%)\end{array}$ & $\begin{array}{c}4 \times 8 \\
(7-94 \%)\end{array}$ & $\begin{array}{c}4 \times 8-10 \mathrm{~kg} \\
(9-90 \%)\end{array}$ & & 2 & $3 \times 15(6-90 \%)$ & $3 \times 15(5-93 \%)$ & $3 \times 12(8-90 \%)$ & $3 \times 8(9-89 \%)$ \\
\hline July-September 2016 & 2 & $\begin{array}{c}4 \times 8-15 \mathrm{~kg} \\
(8-92 \%)\end{array}$ & $\begin{array}{c}4 \times 8-50 / 60 \mathrm{~kg} \\
(7-95 \%)\end{array}$ & $\begin{array}{c}4 \times 8-25 \mathrm{~kg} \\
(7-88 \%)\end{array}$ & $\begin{array}{c}4 \times 8 \\
(7-94 \%)\end{array}$ & $\begin{array}{c}4 \times 8-10 \mathrm{~kg} \\
(8-90 \%)\end{array}$ & & 2 & $3 \times 15(5-90 \%)$ & $3 \times 15(5-94 \%)$ & $3 \times 12(7-88 \%)$ & $3 \times 8(9-88 \%)$ \\
\hline October-December 2016 & \multicolumn{12}{|c|}{ Decrease in medication (corticosteroids) } \\
\hline January-March 2017 & 2 & $\begin{array}{c}4 \times 8-15 \mathrm{~kg} \\
(8-92 \%)\end{array}$ & $\begin{array}{c}4 \times 8-50 / 60 \mathrm{~kg} \\
(8-95 \%)\end{array}$ & $\begin{array}{c}4 \times 8-25 \mathrm{~kg} \\
(8-85 \%)\end{array}$ & $\begin{array}{c}4 \times 8 \\
(7-94 \%)\end{array}$ & & & 2 & $0 / 3 \times 15(6-90 \%)$ & 0/15 (5-92\%) & $0 / 3 \times 12(8-88 \%)$ & $\begin{array}{l}0 / 3 \times 0 / 8 \\
(9-85 \%)\end{array}$ \\
\hline April 2017 & 2 & $\begin{array}{c}4 \times 8-20 \mathrm{~kg} \\
(9-90 \%)\end{array}$ & $\begin{array}{c}4 \times 8-60 \mathrm{~kg} \\
(8-94 \%)\end{array}$ & $\begin{array}{c}4 \times 8-25 \mathrm{~kg} \\
(8-85 \%)\end{array}$ & $\begin{array}{c}4 \times 8 \\
(7-94 \%)\end{array}$ & $\begin{array}{c}4 \times 8-10 \mathrm{~kg} \\
(8-88 \%)\end{array}$ & & 2 & $3 \times 15(6-90 \%)$ & $15(5-92 \%)$ & $3 \times 12(8-88 \%)$ & \\
\hline May-June 2017 & \multicolumn{12}{|c|}{ Progressive withdrawal of medication } \\
\hline July-September 2017 & $0-2$ & $\begin{array}{c}4 \times 8-20 \mathrm{~kg} \\
(8-90 \%)\end{array}$ & $\begin{array}{c}4 \times 8-60 \mathrm{~kg} \\
(7-94 \%)\end{array}$ & $\begin{array}{c}4 \times 8-25 \mathrm{~kg} \\
(8-85 \%)\end{array}$ & $\begin{array}{c}4 \times 8 \\
(7-95 \%)\end{array}$ & $\begin{array}{c}4 \times 8-10 \mathrm{~kg} \\
(8-88 \%)\end{array}$ & & $0-2$ & $0 / 3 \times 12(8-88 \%)$ & $0 / 3 \times 12(9-88 \%)$ & $0 / 3 \times 10(9-88 \%)$ & \\
\hline October-December 2017 & \multicolumn{12}{|c|}{ Exacerbation of sarcoidosis } \\
\hline January-March 2018 & 2 & $\begin{array}{c}4 \times 8-20 / 15 \mathrm{~kg} \\
(7-90 \%)\end{array}$ & $\begin{array}{c}4 \times 8-60 / 70 \mathrm{~kg} \\
(7-94 \%)\end{array}$ & $\begin{array}{c}4 \times 8-20 \mathrm{~kg} \\
(7-89 \%)\end{array}$ & $\begin{array}{c}4 \times 8 \\
(7-95 \%)\end{array}$ & $\begin{array}{c}4 \times 8-10 \mathrm{~kg} \\
(8-90 \%)\end{array}$ & $\begin{array}{c}4 \times 6-7.5 \mathrm{~kg} \\
(9-88 \%)\end{array}$ & 2 & $3 \times 15(6-94 \%)$ & $3 \times 15(5-94 \%)$ & $3 \times 12(6-88 \%)$ & \\
\hline April-June 2018 & 2 & $\begin{array}{c}4 \times 8-15 \mathrm{~kg} \\
(8-88 \%)\end{array}$ & $\begin{array}{c}4 \times 8-60 \mathrm{~kg} \\
(7-94 \%)\end{array}$ & $\begin{array}{c}4 \times 8-20 \mathrm{~kg} \\
(7-89 \%)\end{array}$ & $\begin{array}{c}4 \times 8 \\
(7-95 \%)\end{array}$ & $\begin{array}{c}4 \times 8-8 \mathrm{~kg} \\
(8-89 \%)\end{array}$ & $\begin{array}{c}4 \times 6-7.5 \mathrm{~kg} \\
(9-88 \%)\end{array}$ & 2 & $3 \times 15(5-94 \%)$ & $2 \times 15(5-94 \%)$ & $2 \times 12(5-90 \%)$ & \\
\hline July-September 2018 & $0-2$ & $\begin{array}{c}2 / 4 \times 8-20 \mathrm{~kg} \\
(7-92 \%)\end{array}$ & $\begin{array}{c}4 \times 8-70 \mathrm{~kg} \\
(7-92 \%)\end{array}$ & $\begin{array}{c}2 / 4 \times 8-25 \mathrm{~kg} \\
(8-88 \%)\end{array}$ & $\begin{array}{l}2 / 4 \times 8 \\
(7-95 \%)\end{array}$ & $\begin{array}{c}2 / 4 \times 8-8 \mathrm{~kg} \\
(7-92 \%)\end{array}$ & $\begin{array}{c}4 \mathrm{v} 6-7.5 \mathrm{~kg} \\
(9-88 \%)\end{array}$ & $1-2$ & $3 \times 15(5-94 \%)$ & $0 / 2 \times 15(5-94 \%)$ & $0 / 2 \times 12(5-90 \%)$ & \\
\hline October-December 2018 & $0-2$ & $\begin{array}{c}2 \times 8-20 \mathrm{~kg} \\
(7-92 \%)\end{array}$ & $\begin{array}{c}4 \times 8-70 \mathrm{~kg} \\
(7-94 \%)\end{array}$ & $\begin{array}{c}2 \times 8-20 \mathrm{~kg} \\
(7-88 \%)\end{array}$ & $\begin{array}{c}2 \times 8 \\
(7-95 \%)\end{array}$ & $\begin{array}{c}2 \times 8-8 \mathrm{~kg} \\
(7-92 \%)\end{array}$ & $\begin{array}{c}4 \times 6-7.5 \mathrm{~kg} \\
(9-86 \%)\end{array}$ & $1-2$ & $3 \times 15(5-94 \%)$ & $0 / 3 \times 15(5-95 \%)$ & $0 / 3 \times 12(5-90 \%)$ & $\begin{array}{l}0 / 3 \times 10 \\
(9-85 \%) \\
\end{array}$ \\
\hline January-March 2019 & $1-2$ & $\begin{array}{c}2 / 3 \times 8-20 \mathrm{~kg} \\
(9-88 \%)\end{array}$ & $\begin{array}{c}2 / 3 \times 8-70 \mathrm{~kg} \\
(9-93 \%)\end{array}$ & $\begin{array}{c}2 / 3 \times 8-25 \mathrm{~kg} \\
(9-88 \%)\end{array}$ & $\begin{array}{l}2 / 3 \times 8 \\
(8-95 \%)\end{array}$ & $\begin{array}{c}2 / 3 \times 8-8 \mathrm{~kg} \\
(8-90 \%)\end{array}$ & \multicolumn{6}{|c|}{ Plantar fasciitis } \\
\hline April-June 2019 & 1 & $\begin{array}{c}2 / 3 \times 8-20 \mathrm{~kg} \\
(9-88 \%)\end{array}$ & $\begin{array}{c}2 / 3 \times 8-70 \mathrm{~kg} \\
(8-93 \%)\end{array}$ & $\begin{array}{c}2 / 4 \times 8-25 \mathrm{~kg} \\
(9-85 \%)\end{array}$ & $\begin{array}{l}2 / 4 \times 8 \\
(8-93 \%)\end{array}$ & $\begin{array}{c}2 / 3 \times 8-8 \mathrm{~kg} \\
(9-90 \%)\end{array}$ & & 1 & $3 \times 15(8-90 \%)$ & $3 \times 15(8-92 \%)$ & $3 \times 12(7-90 \%)$ & 4 static planks \\
\hline July-September 2019 & 1 & $\begin{array}{c}4 \times 8-20 \mathrm{~kg} \\
(8-92 \%)\end{array}$ & $\begin{array}{c}4 \times 8-70 \mathrm{~kg} \\
(7-94 \%)\end{array}$ & $\begin{array}{c}4 \times 8-25 \mathrm{~kg} \\
(7-85 \%)\end{array}$ & $\begin{array}{c}4 \times 8 \\
(9-88 \%)\end{array}$ & & & 1 & $3 \times 15(8-90 \%)$ & $3 \times 15(8-90 \%)$ & $3 \times 12(6-90 \%)$ & 4 static planks \\
\hline October-December 2019 & 1 & $\begin{array}{c}4 \times 8-20 \mathrm{~kg} \\
(7-92 \%) \\
\end{array}$ & $\begin{array}{c}4 \times 8-70 \mathrm{~kg} \\
(7-95 \%) \\
\end{array}$ & $\begin{array}{c}4 \times 8-25 \mathrm{~kg} \\
(7-85 \%) \\
\end{array}$ & $\begin{array}{c}4 \times 8 \\
(9-88 \%) \\
\end{array}$ & & & 1 & $3 \times 15(9-90 \%)$ & $3 \times 15(8-90 \%)$ & $3 \times 12(6-90 \%)$ & 4 static planks \\
\hline
\end{tabular}


Thereafter, eccentric Russian belt squat (Video S3) and, also, clean and jerk exercises were added to the gym machine routine. From the seventh month, and on different days, a functional exercise routine (step-full squats (Video S4), lunges (Video S5), step-squat jumps (Video S6), and burpees (Video S7)) was added and performed twice weekly (Table 2). $\mathrm{HR}$ and $\mathrm{SpO}_{2}$ were continuously monitored in all sessions, as was RPE, using the 0-10 OMNI Resistance Exercise Scale [29] at the end of each exercise. The resistance training scheme aimed to allow the patient to achieve the highest maximum training volume, combining high loads and low repetitions while keeping $\mathrm{SpO}_{2}$ above $90 \%$ (or at least allowing a rapid recovery of this variable to baseline levels in case it fell below 90\%). This combination was selected since high loads increase maximum force more than low loads [30], and a low number of sets and repetitions have been shown as effective in increasing strength as a combination of a high number of sets and repetitions in post-menopausal women [31].

IMT was done using a mechanical pressure threshold device (Powerbreathe Plus Low Resistance, Powerbreathe Spain-Biocorp Europa; Andoain, Spain). It started with two sessions (morning and evening) of 10 maximum inspirations at $42 \%$ of MIP, five days a week, increasing gradually to 30 inspirations per session in the sixth month. Thereafter, the number of repetitions was maintained, and the inspiratory load was progressively increased depending on the patient's intensity perception. She was asked to gradually increase the load of the inspiratory device so that it was always difficult, but not impossible, for her to do all the inspirations. After the second year, she performed two sessions of 60 inspirations per day, increasing the inspiratory load as described.

\section{Results}

During the study period the patient's functional status improved from NYHA III (at the start of the intervention, in the middle of year 2015) to II (at the conclusion of the 4.5-year intervention, end of year 2019). At the end of 2016 and throughout 2017 several breaks in training occurred due to exacerbation of the disease likely associated with the progressive reduction in corticoid dosage (since clinical severity had decreased) until the withdrawal of the drug in May 2017 and reintroduction again at the start of 2018 (due to exacerbation of symptoms) (Figure 1). In addition, during January-March 2019, the patient developed plantar fasciitis that led to the suspension of HIIT during this period. Although in some months $\mathrm{SpO}_{2}$ dropped below $90 \%$ after the HIIT sprints, it consistently returned to baseline levels very quickly (within 10-15 s).

Strength training was affected by medication withdrawal in the same way as HIIT (Table 2). Plantar fasciitis forced the suspension of the dynamic exercises, and the replacement of burpees with static planks. However, machine strength training was maintained throughout the duration of injury. As for the HIIT sessions, in those cases where $\mathrm{SpO}_{2}$ dropped below $90 \%$ it normalized very quickly (10-15 s).

The inspiratory load increased from 20 to 40 inspirations per day at $42 \%$ MIP of restriction to 120 inspirations per day at $85 \%$ of MIP, causing a $19 \%$ increase in MIP (reaching $127 \mathrm{~cm} \mathrm{H}_{2} \mathrm{O}$ ). In addition to the interruptions of IMT due to the worsening of her condition after the medication withdrawal, IMT was suspended in March 2019 due to rib pain after a fall while running on the treadmill.

The effects of training in cardiopulmonary exercise testing data are shown in Table 3. Notably, $\mathrm{VO}_{2 \text { peak }}$ and peak pulmonary ventilation increased by $44 \%$ and $60 \%$, respectively, from the start to the end of the study period. Table 4 summarizes total and regional bone mineral density (BMD), lean and fat mass values during the study. 
Table 3. Results of cardiopulmonary exercise testing.

\begin{tabular}{|c|c|c|c|c|c|c|c|c|c|c|c|}
\hline Variables & Pretraining & $\begin{array}{c}\text { December } \\
2015\end{array}$ & June 2016 & $\begin{array}{c}\text { December } \\
2016\end{array}$ & June 2017 & $\begin{array}{c}\text { December } \\
2017\end{array}$ & June 2018 & $\begin{array}{c}\text { December } \\
2018\end{array}$ & June 2019 & $\begin{array}{c}\text { December } \\
2019\end{array}$ & $\begin{array}{c}\text { Change from } \\
\text { Baseline to } 4.5 \text { Years } \\
\text { Later }(\%)\end{array}$ \\
\hline \multicolumn{12}{|l|}{ Peak values } \\
\hline $\begin{array}{l}\mathrm{VO}_{2} \text { peak } \\
\left(\mathrm{mL} \cdot \mathrm{kg}^{-1} \cdot \mathrm{min}^{-1}\right)\end{array}$ & 20.1 & 22.4 & 30.2 & 23.6 & 24.4 & 25.2 & 28.9 & 28.3 & 26.6 & 28.9 & $+44 \%$ \\
\hline $\begin{array}{l}\mathrm{VO}_{2} \text { peak } \\
\left(\mathrm{mL} \cdot \mathrm{min}^{-1}\right)\end{array}$ & 1086 & 1212 & 1631 & 1275 & 1316 & 1360 & 1560 & 1550 & 1502 & 1561 & $+44 \%$ \\
\hline PPO (watts) & 100 & 119 & 123 & 130 & 125 & 120 & 130 & 127 & 130 & 124 & $+24 \%$ \\
\hline HRpeak (bpm) & 166 & 164 & 164 & 167 & 162 & 170 & 170 & 166 & 170 & 172 & $+4 \%$ \\
\hline HR (\% HRmax) & 99 & 98 & 98 & 100 & 98 & 102 & 103 & 100 & 104 & 105 & $+6 \%$ \\
\hline VEpeak (L/min) & 62 & 59 & 73 & 65 & 83 & 87 & 81 & 97 & 77 & 99 & $+60 \%$ \\
\hline $\mathrm{SpO}_{2}$ peak $(\%)$ & 93 & 91 & 91 & 88 & 89 & 88 & 89 & 89 & 89 & 91 & $-2 \%$ \\
\hline \multicolumn{12}{|l|}{ VT } \\
\hline $\begin{array}{l}\mathrm{VO}_{2} \\
\left(\mathrm{~mL}^{2} \mathrm{~kg}^{-1} \cdot \mathrm{min}^{-1}\right)\end{array}$ & 11.7 & 14.2 & 18.7 & 11.2 & 12.8 & 15.4 & 17.9 & 11.7 & 15.6 & 15.1 & $+29 \%$ \\
\hline $\mathrm{PO}$ (watts) & 47 & 57 & 66 & 35 & 47 & 50 & 47 & 41 & 60 & 42 & $-11 \%$ \\
\hline $\mathrm{VE} \cdot \mathrm{VO}_{2}{ }^{-1}$ & 38 & 29 & 31 & 28 & 31 & 27 & 29 & 25 & 28 & 26 & $-32 \%$ \\
\hline $\mathrm{VE} \cdot \mathrm{VCO}_{2}{ }^{-1}$ & 39 & 39 & 35 & 35 & 39 & 33 & 38 & 35 & 34 & 38 & $-3 \%$ \\
\hline \multicolumn{12}{|l|}{ RCP } \\
\hline $\begin{array}{l}\mathrm{VO}_{2} \\
\left(\mathrm{~mL} \mathrm{~kg}^{-1} \cdot \mathrm{min}^{-1}\right)\end{array}$ & 17.4 & 18.1 & 26.6 & 19.8 & 19.3 & 21.2 & 24.1 & 22.3 & 18.3 & 23.9 & $+37 \%$ \\
\hline $\mathrm{PO}$ (watts) & 77 & 87 & 103 & 106 & 89 & 87 & 83 & 102 & 95 & 90 & $+17 \%$ \\
\hline $\mathrm{VE} \cdot \mathrm{VO}_{2}{ }^{-1}$ & 42 & 42 & 35 & 36 & 36 & 35 & 35 & 36 & 32 & 36 & $-14 \%$ \\
\hline $\mathrm{VE} \cdot \mathrm{VCO}_{2}{ }^{-1}$ & 36 & 37 & 33 & 32 & 35 & 32 & 36 & 32 & 33 & 37 & $+3 \%$ \\
\hline
\end{tabular}

Abbreviations: $\mathrm{HR}$, heart rate; $\mathrm{HRmax}$ (age-predicted maximum heart rate, i.e., 220 minus age, in years); $\mathrm{HRpeak}$, peak heart rate; $\mathrm{PO}$, power output; $\mathrm{PPO}$, peak power output; $\mathrm{RCP}$ respiratory compensation point; $\mathrm{SpO}_{2}$, peripheral capillary oxygen saturation; $\mathrm{VE}^{-\mathrm{VO}_{2}}{ }^{-1}$, ventilatory equivalent for oxygen. $\mathrm{VE} \cdot \mathrm{VCO}_{2}{ }^{-1}$, ventilatory equivalent for carbon dioxide. VEpeak, peak ventilation; $\mathrm{VO}_{2}$ : oxygen uptake; $\mathrm{VO}_{2}$ peak, peak oxygen uptake; $\mathrm{VT}$, ventilatory threshold. 
Table 4. Results of body composition.

\begin{tabular}{|c|c|c|c|c|c|c|c|c|c|c|}
\hline Variables & $\begin{array}{l}\text { Oct. } \\
2007\end{array}$ & $\begin{array}{l}\text { June } \\
2016\end{array}$ & $\begin{array}{c}\text { December } \\
2016\end{array}$ & $\begin{array}{l}\text { June } \\
2017\end{array}$ & $\begin{array}{c}\text { December } \\
2017\end{array}$ & $\begin{array}{l}\text { June } \\
2018\end{array}$ & $\begin{array}{c}\text { December } \\
2018\end{array}$ & $\begin{array}{l}\text { June } \\
2019\end{array}$ & $\begin{array}{c}\text { December } \\
2019\end{array}$ & $\begin{array}{l}\text { Change from Baseline } \\
\text { to } 3.5 \text { Years Later }(\%)\end{array}$ \\
\hline \multicolumn{11}{|l|}{$\operatorname{BMD}\left(\mathrm{g} \cdot \mathrm{cm}^{-2}\right)$} \\
\hline Whole body & & 1.11 & 1.07 & 1.07 & 1.05 & 1.06 & 1.04 & 1.02 & 1.06 & -4.5 \\
\hline Subtotal body & & 0.91 & 0.89 & 0.89 & 0.87 & 0.88 & 0.86 & 0.86 & 0.89 & -2.2 \\
\hline Pelvic & & 1.09 & 1.06 & 1.06 & 1.06 & 1.07 & 1.01 & 1.05 & 1.10 & 0.9 \\
\hline Arms (mean) & & 0.67 & 0.65 & 0.65 & 0.64 & 0.65 & 0.65 & 0.64 & 0.65 & -3.0 \\
\hline Legs (mean) & & 1.10 & 1.09 & 1.08 & 1.06 & 1.04 & 1.06 & 1.06 & 1.06 & -3.6 \\
\hline Lumbar (mean $\left.\mathrm{L}_{1}-\mathrm{L}_{4}\right)$ & 1.24 & 0.98 & 0.97 & 0.96 & 0.94 & 0.92 & 0.92 & 0.93 & 0.96 & -2.0 \\
\hline T-Score spine & 0.4 & -0.6 & -0.7 & -0.8 & -0.9 & -1.2 & -1.1 & -1.1 & -0.8 & \\
\hline Z-Score spine & 1.1 & 0.4 & 0.3 & 0.3 & 0.1 & -0.1 & 0.0 & 0.1 & 0.4 & \\
\hline Femoral neck & 1.01 & 0.83 & 0.76 & 0.81 & 0.84 & 0.76 & 0.73 & 0.74 & 0.73 & -12.0 \\
\hline T-Score femoral neck & 0.2 & -0.1 & -0.8 & -0.3 & -0.1 & -0.8 & -1.2 & -1.0 & -1.1 & \\
\hline Z-Score femoral neck & 0.7 & 0.8 & 0.2 & 0.7 & 1.0 & 0.2 & 0.1 & 0.1 & 0.1 & \\
\hline \multicolumn{11}{|l|}{ Lean mass $(\mathrm{kg})$} \\
\hline Whole body & & 40.4 & 38.5 & 39.6 & 38.5 & 40.4 & 39.2 & 38.4 & 35.8 & -11.4 \\
\hline Subtotal body & & 35.6 & 33.6 & 34.6 & 33.7 & 35.5 & 34.4 & 35.4 & 33.0 & -7.3 \\
\hline Trunk & & 19.0 & 17.7 & 18.6 & 17.5 & 18.6 & 18.9 & 19.4 & 17.2 & -9.5 \\
\hline Arms (mean) & & 2.0 & 1.9 & 2.0 & 1.9 & 2.1 & 1.9 & 1.8 & 1.9 & -5.0 \\
\hline Legs (mean) & & 6.3 & 6.1 & 6.1 & 6.2 & 6.4 & 5.9 & 6.3 & 6.0 & -4.8 \\
\hline \multicolumn{11}{|l|}{ Fat mass $(\mathrm{kg})$} \\
\hline Whole body & & 10.3 & 12.9 & 12.9 & 12.1 & 13.9 & 14.1 & 16.3 & 15.9 & 54.4 \\
\hline Subtotal body & & 9.6 & 12.2 & 12.1 & 11.4 & 13.2 & 13.3 & 15.6 & 15.2 & 58.3 \\
\hline Trunk & & 3.7 & 4.9 & 4.7 & 4.6 & 5.3 & 5.4 & 6.2 & 6.3 & 70.3 \\
\hline Arms (mean) & & 0.3 & 0.4 & 0.4 & 0.4 & 0.5 & 0.5 & 0.7 & 0.6 & 100 \\
\hline Legs (mean) & & 2.6 & 3.2 & 3.3 & 3.0 & 3.5 & 3.4 & 4.0 & 3.9 & 50 \\
\hline Fat mass (\%) & & 20.3 & 26.0 & 24.6 & 23.9 & 25.6 & 26.4 & 28.8 & 29.7 & 46.3 \\
\hline
\end{tabular}




\section{Discussion}

The main finding of our study was that a combined (HIIT + strength + IMT) program, applied for 4.5 years in a patient with sarcoidosis (stage IV) and pulmonary involvement and treated with corticoids for 12 years, contributed - at least partly - to increase functional capacity (improving a class in the NYHA classification) as well as cardiorespiratory fitness (CRF, i.e., increase in $\mathrm{VO}_{2}$ peak from barely $\sim 6$ metabolic equivalents (MET) at the start of the study to $8.3 \mathrm{MET}$ at the end). This is an important finding because the $\mathrm{VO}_{2}$ peak level reflects the synergistic action of pulmonary, cardiovascular and muscle tissue via a suite of physiological actions that effectively transport and deliver oxygen from the atmosphere to mitochondria in working muscles [32]. CRF, as determined by $\mathrm{VO}_{2}$ peak, is a strong prognostic factor of morbimortality, particularly in relation to cardiometabolic diseases [33]. Moreover, it is of clinical importance to surpass the eight-MET threshold, and in fact adults with a $\mathrm{VO}_{2}$ peak above this level have a reduced cardiovascular risk [34]. Of note, the increase in CRF was corroborated-being in fact of the same relative magnitude-when $\mathrm{VO}_{2}$ peak was expressed in either absolute units $\left(\mathrm{mL} \cdot \mathrm{min}^{-1}\right)$ or relative to body mass $\left(\mathrm{mL} \cdot \mathrm{kg}^{-1} \cdot \mathrm{min}^{-1}\right)$. Furthermore, the intervention was safe, with no persistent $\mathrm{SpO}_{2}$ drops or syncope episodes.

A reduced maximum exercise capacity is related to frailty, conceptually defined as a physical vulnerability to stressors. Frailty is considered an important phenotype in lung transplant candidates independent of the severity of the disease [35]. On the other hand, 10\% of patients with sarcoidosis progress to fibrotic lung disease and may be in need of lung transplant [18]. In this regard, although lung transplantation was originally proposed as a future option for our patient, the remission of right atrium dilatation, mild mitral insufficiency, and PAH, together with improvements in NYHA class and CRF made it possible to discard transplant by the end of first year of the intervention.

HIIT induces both peripheral (increased muscle capillary density and mitochondrial content) and central (increased cardiac output) cardiovascular adaptations [36]. Furthermore, HIIT can increase alveolar capillary density through nitric oxide-induced vascular endothelial growth factor (VEGF) [37]. In fact, the HIIT protocol used here was designed to allow the accumulation of as many training loads near $\mathrm{VO}_{2}$ peak as possible without drops in $\mathrm{SpO}_{2}$. One the one hand, and likely related to this, we observed an improvement in ventilatory efficiency, as expressed in $\mathrm{VE} \cdot \mathrm{VO}_{2}{ }^{-1}$ at submaximal intensities (which decreased by $32 \%$ and $14 \%$ at VT and RCP, respectively, with the latter value similar to that of healthy subjects) [38]. Improved ventilatory efficiency might have contributed, at least in part, to the observed increase in the patient's functional independence and the absence of high $\mathrm{SpO}_{2}$ drops in her daily life activities.

IMT has been shown to improve exercise capacity, functionality, respiratory muscle strength, dyspnea, and fatigue in patients with sarcoidosis [39]. These improvements can be explained by the resulting increase in respiratory muscle strength [40] and are reflected by increases in MIP and peak ventilation during exercise. IMT-induced improvements in lung compliance can increase the surface of gas exchange [41]. Furthermore, IMT decreases the oxygen cost of breathing [41], thereby reducing the fatigue-induced respiratory metabolic reflex with subsequent increases in blood flow to locomotor muscles [42].

Resistance training may also have played a role in increasing the $\mathrm{VO}_{2}$ peak ( $\mathrm{mL} / \mathrm{min}$ ) and improving the patient's NYHA functional class. The increase in maximum strength may have allowed her, at least partly, to maintain a higher training work rate and increase the ability to cope with-and adapt to-cumulative HIIT sessions. Resistance training is known to improve work economy [43] and thus decreases the percentage of work demanded by daily life activities (e.g., walking, climbing stairs, and carrying bags); it thus allows performing these submaximal actions with less oxygen cost [44]. Muscle mass could have been affected by the patient's menopause and prednisone treatment. Menopause frequently causes sarcopenia, which is a syndrome characterized by a progressive and generalized loss of skeletal muscle mass and strength [45]. Likewise, corticoid treatment reduces protein synthesis and increases muscle protein catabolism [46], especially in type II fibers [47]. Although muscle mass contributes to improvements in strength, our approach was actually designed to improve 
muscle strength through neural factors, by increasing motor unit recruitment and firing frequency of active motor units without the need for major increases in muscle mass [48]. This is in fact why high loads were used, as they increase neural adaptations compared with lower loads [49]. In this regard, a previous study applied high-load strength training to patients with sarcoidosis for 12 weeks [13]. Although the relatively short training duration of that study suggests that the strength gains noted were likely due to neural factors, the absence of muscle mass measurements and the persistence of adaptations five months after the training period makes it difficult to quantify the neural factor influence. Our results are, however, in line with the Kullberg et al. study regarding the feasibility, safety and effectiveness of high-load resistance training in patients with sarcoidosis.

It is widely known that bone and lean mass decrease with age, particularly in menopausal women due to hormonal changes [50]. Corticoids also accelerate the loss of bone mass [51] whereas the opposite effect is seen with exercise, which is in fact recommended for the prevention of osteoporosis and osteopenia [52]. Our results would support the beneficial effect of exercise on bone health, since we observed only a slight decrease in BMD over the three-year period despite the post-menopausal status of the patient and the corticoid treatment she received for years. Indeed, analysis at the end of the follow-up revealed only mild osteopenia of the femoral neck and normal bone health at the spine (T-score $=-1.1$ and -0.8 , respectively), indicating that our patient was above the average for women of her age (Z-score range $=0.1-0.4$ ). Similarly, the exercise intervention probably had a certain beneficial effect on lean mass, which decreased only in the last measurement likely due to the reduction in the volume of strength training, which dropped from 4 to 2 days/week after a plantar fasciitis.

The NYHA class improvement, together with the well-known deleterious effects of chronic corticoid treatment led to the progressive withdrawal of this drug after 1.5 years from the start of the training program. With the exception of the sarcoidosis flare-up caused by medication withdrawal, only one sarcoidosis-related hospital admission (due to respiratory infection) and one medical intervention (capsulotomy) related to sarcoidosis occurred in the 4.5 years. Adverse events related to training were a running fall from the treadmill (not associated with arterial oxygen desaturation) and plantar fasciitis. Despite these episodes, the patient maintained her adherence to the training program due to the positive impact that the improvement in QoL and wellbeing early during the intervention (in the first few months) had on her level of motivation, as well as to her strong self-discipline.

The present study has several limitations that should be considered. First, as this is a case report, our preliminary results in only one patient must be interpreted with caution and cannot be generalized to all patients with sarcoidosis. In this regard, it would be interesting to conduct a randomized controlled trial with a long-term intervention like the present one, although this type of study might be very difficult to conduct. Second, the 1RM was not evaluated, and thus although an increase in load $(\mathrm{kg})$ was observed with resistance training, we could not quantify the magnitude of the actual improvement in maximum strength. Finally, diet was not controlled.

\section{Conclusions}

In summary, the results of this study suggest that a long-term combined (HIIT + strength + IMT) exercise intervention might have contributed, at least partly, to safely improve CRF and functional capacity (NYHA class) in a patient with severe thoracic sarcoidosis. Our results also suggest that the intervention could have acted as a coadjuvant treatment that helped to partially reduce the severity of associated pathologies (cardiac involvement and PAH) and attenuate the loss of BMD and muscle mass caused by menopause and chronic corticoid treatment. Randomized controlled trials are needed to confirm our preliminary findings.

Supplementary Materials: The following are available online at http://www.mdpi.com/1660-4601/17/24/9512/s1, Video S1: Modified Chest Fly exercise, Video S2: Modified Lat Pull Down exercise, Video S3: Russian Belt Squat exercise, Video S4: Step-full squat exercise, Video S5: Lunge exercise, Video S6: Step-squat jump exercise, Video S7: Burpee exercise. 
Author Contributions: Conceptualization, J.M.G.-M., A.S., and A.L.; methodology, J.M.G.-M., A.S., I.R.-G., I.A., and A.M.H.-O.; formal analysis, A.S., I.R.-G., I.A., and A.M.H.-O.; data curation, I.R.-G., I.A., and A.M.H.-O.; writing—original draft preparation, A.M.H.-O.; writing—review and editing, A.S., I.A., and A.L.; visualization, A.S. and A.L.; supervision, A.S. and A.L. All authors have read and agreed to the published version of the manuscript.

Funding: This Research by A.L. is funded by the Spanish Ministry of Science and Innovation (Fondo de Investigaciones Sanitarias) and Fondos Feder, grant number PI18/00139. Irene Rodríguez Gómez received a postdoctoral contract from the Government of Castilla-La Mancha (2019/9601) and Alba M. Herrera Olivares received a predoctoral contract from Spanish Ministry of Science, Innovation and Universities (FPU18/00215). Research by A.L. is supported by a grant from Spanish Instituto de Salud Carlos III (ISCIII)-ERDF (PI18/00139). The Biomedical Research Networking Center on Frailty and Healthy Aging (CIBERFES) and FEDER funds from the European Union (CB16/10/00477 and CB16/10/00314).

Conflicts of Interest: The authors declare no conflict of interests.

\section{References}

1. Arkema, E.V.; Cozier, Y.C. Epidemiology of sarcoidosis: Current findings and future directions. Ther. Adv. Chronic Dis. 2018, 9, 227-240. [CrossRef] [PubMed]

2. Tavana, S.; Alizadeh, M.; Mohajerani, S.; Hashemian, S. Pulmonary and extra-pulmonary manifestations of sarcoidosis. Niger. Med. J. 2015, 56, 258. [CrossRef] [PubMed]

3. Voortman, M.; Hendriks, C.M.R.; Elfferich, M.D.P.; Bonella, F.; Møller, J.; de Vries, J.; Costabel, U.; Drent, M. The burden of sarcoidosis symptoms from a patient perspective. Lung 2019, 197, 155-161. [CrossRef] [PubMed]

4. Judson, M.A. The clinical features of sarcoidosis: A comprehensive review. Clin. Rev. Allergy Immunol. 2015, 49, 63-78. [CrossRef] [PubMed]

5. Boucly, A.; Cottin, V.; Nunes, H.; Jaïs, X.; Tazi, A.; Prévôt, G.; Reynaud-Gaubert, M.; Dromer, C.; Viacroze, C.; Horeau-Langlard, D.; et al. Management and long-term outcomes of sarcoidosis-associated pulmonary hypertension. Eur. Respir. J. 2017, 50. [CrossRef]

6. Moor, C.C.; Gür-Demirel, Y.; Wijsenbeek, M.S. Feasibility of a comprehensive home monitoring program for sarcoidosis. J. Personal. Med. 2019, 9, 23. [CrossRef]

7. Cho, P.S.P.; Vasudevan, S.; Maddocks, M.; Spinou, A.; Mitchell, S.C.; Wood, C.; Jolley, C.J.; Birring, S.S. Physical inactivity in pulmonary sarcoidosis. Lung 2019, 197, 285-293. [CrossRef]

8. Froidure, S.; Kyheng, M.; Grosbois, J.M.; Lhuissier, F.; Stelianides, S.; Wemeau, L.; Wallaert, B. Daily life physical activity in patients with chronic stage IV sarcoidosis: A multicenter cohort study. Health Sci. Rep. 2019, 2, e109. [CrossRef]

9. Bahmer, T.; Watz, H.; Develaska, M.; Waschki, B.; Rabe, K.F.; Magnussen, H.; Kirsten, D.; Kirsten, A.-M. Physical activity and fatigue in patients with sarcoidosis. Respiration 2018, 95, 18-26. [CrossRef]

10. Strookappe, B.; Saketkoo, L.A.; Elfferich, M.; Holland, A.; de Vries, J.; Knevel, T.; Drent, M. Physical activity and training in sarcoidosis: Review and experience-based recommendations. Expert Rev. Respir. Med. 2016, 10, 1057-1068. [CrossRef]

11. Strookappe, B.; Elfferich, M.; Swigris, J.; Verschoof, A.; Veschakelen, J.; Knevel, T.; Drent, M. Benefits of physical training in patients with idiopathic or end-stage sarcoidosis-related pulmonary fibrosis: A pilot study. Sarcoidosis Vasc. Diffuse Lung Dis. 2015, 32, 43-52. [PubMed]

12. Marcellis, R.; van der Veeke, M.; Mesters, I.; Drent, M.; de Bie, R.; de Vries, G.; Lenssen, A. Does physical training reduce fatigue in sarcoidosis? Sarcoidosis Vasc. Diffuse Lung Dis. 2015, 32, 53-62. [PubMed]

13. Kullberg, S.; Rivera, N.; Eriksson, M.J.; Grunewald, J.; Eklund, A. High-intensity resistance training in newly diagnosed sarcoidosis- an exploratory study of effects on lung function, muscle strength, fatigue, dyspnea, health-related quality of life and lung immune cells. Eur. Clin. Respir. J. 2020, 7, 1730137. [CrossRef] [PubMed]

14. Strookappe, B.; Swigris, J.; de Vries, J.; Elfferich, M.; Knevel, T.; Drent, M. Benefits of physical training in sarcoidosis. Lung 2015, 193, 701-708. [CrossRef] [PubMed]

15. Naz, I.; Ozalevli, S.; Ozkan, S.; Sahin, H. Efficacy of a structured exercise program for improving functional capacity and quality of life in patients with stage 3 and 4 sarcoidosis: A randomized controlled trial. J. Cardiopulm. Rehabil. Prev. 2018, 38, 124-130. [CrossRef] [PubMed] 
16. Gibala, M.J.; Little, J.P.; Macdonald, M.J.; Hawley, J.A. Physiological adaptations to low-volume, high-intensity interval training in health and disease. J. Physiol. 2012, 590, 1077-1084. [CrossRef] [PubMed]

17. Grongstad, A.; Vøllestad, N.; Oldervoll, L.; Spruit, M.; Edvardsen, A. The effects of high- versus moderate-intensity exercise on fatigue in sarcoidosis. J. Clin. Med. 2019, 8, 460. [CrossRef]

18. Gangemi, A.; Myers, C.N.; Zheng, M.; Brown, J.; Butler-Lebair, M.; Cordova, F.; Marchetti, N.; Criner, G.J.; Gupta, R.; Mamary, A.J. Mortality for sarcoidosis patients on the transplant wait list in the Lung Allocation Score era: Experience from a high volume center. Respir. Med. 2019, 157, 69-76. [CrossRef]

19. Marcellis, R.G.J.; Lenssen, A.F.; de Vries, J.; Drent, M. Reduced muscle strength, exercise intolerance and disabling symptoms in sarcoidosis. Curr. Opin. Pulm. Med. 2013, 19, 524-530. [CrossRef]

20. Brancaleone, P.; Perez, T.; Robin, S.; Neviere, R.; Wallaert, B. Clinical impact of inspiratory muscle impairment in sarcoidosis. Sarcoidosis Vasc. Diffuse Lung Dis. 2004, 21, 219-227.

21. Hunninghake, G.W.; Costabel, U.; Ando, M.; Baughman, R.; Cordier, J.F.; Bois, R.; Eklund, A.; Kitaichi, M.; Lynch, J.; Rizzto, G.; et al. Statement on sarcoidosis. Am. J. Respir. Crit. Care Med. 1999, 160, 736-755. [CrossRef]

22. González-Saiz, L.; Fiuza-Luces, C.; Sanchis-Gomar, F.; Santos-Lozano, A.; Quezada-Loaiza, C.A.; Flox-Camacho, A.; Munguía-Izquierdo, D.; Ara, I.; Santalla, A.; Morán, M.; et al. Benefits of skeletal-muscle exercise training in pulmonary arterial hypertension: The WHOLEi +12 trial. Int. J. Cardiol. 2017, 231, 277-283. [CrossRef] [PubMed]

23. Zach, M.S. The physiology of forced expiration. Paediatr. Respir. Rev. 2000, 1, 36-39. [CrossRef] [PubMed]

24. Lopes, A.J.; Menezes, S.L.S.; Dias, C.M.; Oliveira, J.F.; Mainenti, M.R.M.; Guimarães, F.S. Cardiopulmonary exercise testing variables as predictors of long-term outcome in thoracic sarcoidosis. Braz. J. Med. Biol. Res. 2012, 45, 256-263. [CrossRef] [PubMed]

25. Lucía, A.; Hoyos, J.; Chicharro, J.L. Preferred pedalling cadence in professional cycling. Med. Sci. Sports Exerc. 2001, 33, 1361-1366. [CrossRef]

26. Rodríguez-Gómez, I.; Santalla, A.; Díez-Bermejo, J.; Munguía-Izquierdo, D.; Alegre, L.M.; Nogales-Gadea, G.; Arenas, J.; Martín, M.A.; Lucia, A.; Ara, I. A new condition in McArdle disease: Poor bone HealthBenefits of an active lifestyle. Med. Sci. Sports Exerc. 2018, 50, 3-10. [CrossRef] [PubMed]

27. Bailey, C.A.; Brooke-Wavell, K. Optimum frequency of exercise for bone health: Randomised controlled trial of a high-impact unilateral intervention. Bone 2010, 46, 1043-1049. [CrossRef]

28. Kemmler, W.; Kohl, M.; von Stengel, S. Long-term effects of exercise in postmenopausal women: 16-year results of the Erlangen Fitness and Osteoporosis Prevention Study (EFOPS). Menopause 2017, 24, 45-51. [CrossRef]

29. Lagally, K.M.; Robertson, R.J. Construct validity of the OMNI resistance exercise scale. J. Strength Cond. Res. 2006, 20, 252-256. [CrossRef]

30. Schoenfeld, B.J.; Grgic, J.; Ogborn, D.; Krieger, J.W. Strength and hypertrophy adaptations between lowvs. High-load resistance training: A systematic review and meta-analysis. J. Strength Cond. Res. 2017, 31, 3508-3523. [CrossRef]

31. Nunes, P.R.; Barcelos, L.C.; Oliveira, A.A.; Furlanetto, R.; Martins, F.M.; Resende, E.A.; Orsatti, F.L.; Júnior, R.F. Muscular strength adaptations and hormonal responses after two different multiple-set protocols of resistance training in postmenopausal women. J. Strength Cond. Res. 2019, 33, 1276-1285. [CrossRef] [PubMed]

32. Valenzuela, P.L.; Maffiuletti, N.A.; Joyner, M.J.; Lucia, A.; Lepers, R. Lifelong endurance exercise as a countermeasure against age-related $\mathrm{V}^{\cdot} \mathrm{O} 2$ max decline: Physiological overview and insights from masters athletes. Sports Med. 2020, 50, 703-716. [CrossRef] [PubMed]

33. Ozemek, C.; Laddu, D.R.; Lavie, C.J.; Claeys, H.; Kaminsky, L.A.; Ross, R.; Wisloff, U.; Arena, R.; Blair, S.N. An update on the role of cardiorespiratory fitness, structured exercise and lifestyle physical activity in preventing cardiovascular disease and health risk. Prog. Cardiovasc. Dis. 2018, 61, 484-490. [CrossRef] [PubMed]

34. Barnes, J.N.; Joyner, M.J. Physical activity and cardiovascular risk: 10 metabolic equivalents or bust. Mayo Clin. Proc. 2013, 88, 1353-1355. [CrossRef] [PubMed]

35. Layton, A.M.; Armstrong, H.F.; Baldwin, M.R.; Podolanczuk, A.J.; Pieszchata, N.M.; Singer, J.P.; Arcasoy, S.M.; Meza, K.S.; D'Ovidio, F.; Lederer, D.J. Frailty and maximal exercise capacity in adult lung transplant candidates. Respir. Med. 2017, 131, 70-76. [CrossRef] [PubMed] 
36. MacInnis, M.J.; Gibala, M.J. Physiological adaptations to interval training and the role of exercise intensity. J. Physiol. 2017, 595, 2915-2930. [CrossRef]

37. Bloor, C.M. Angiogenesis during exercise and training. Angiogenesis 2005, 8, 263-271. [CrossRef] [PubMed]

38. Habedank, D.; Reindl, I.; Vietzke, G.; Bauer, U.; Sperfeld, A.; Wernecke, K.D.; Kleber, F.X. Ventilatory efficiency and exercise tolerance in 101 healthy volunteers. Eur. J. Appl. Physiol. Occup. Physiol. 1998, 77, 421-426. [CrossRef]

39. Karadall, M.N.; Boşnak-Güçlü, M.; Camcıoğlu, B.; Kokturk, N.; Tüktaş, H. Effects of inspiratory muscle training in subjects with sarcoidosis: A randomized controlled clinical trial. Respir. Care 2016, 61, 483-494. [CrossRef]

40. Souza, H.; Rocha, T.; Pessoa, M.; Rattes, C.; Brandão, D.; Fregonezi, G.; Campos, S.; Aliverti, A.; Dornelas, A. Effects of inspiratory muscle training in elderly women on respiratory muscle strength, diaphragm thickness and mobility. J. Gerontol. A Biol. Sci. Med. Sci. 2014, 69, 1545-1553. [CrossRef]

41. Shei, R.J.; Paris, H.L.R.; Wilhite, D.P.; Chapman, R.F.; Mickleborough, T.D. The role of inspiratory muscle training in the management of asthma and exercise-induced bronchoconstriction. Phys. Sportsmed. 2016, 44, 327-334. [CrossRef] [PubMed]

42. Dempsey, J.A.; Romer, L.; Rodman, J.; Miller, J.; Smith, C. Consequences of exercise-induced respiratory muscle work. Respir. Physiol. Neurobiol. 2006, 151, 242-250. [CrossRef] [PubMed]

43. Hoff, J.; Gran, A.; Helgerud, J. Maximal strength training improves aerobic endurance performance. Scand. J. Med. Sci. Sports 2002, 12, 288-295. [CrossRef] [PubMed]

44. Heggelund, J.; Fimland, M.S.; Helgerud, J.; Hoff, J. Maximal strength training improves work economy, rate of force development and maximal strength more than conventional strength training. Eur. J. Appl. Physiol. 2013, 113, 1565-1573. [CrossRef] [PubMed]

45. Ono, Y.; Miyakoshi, N.; Kasukawa, Y.; Akagawa, M.; Kimura, R.; Nagahata, I.; Yuasa, Y.; Sato, C.; Shimada, Y. Diagnosis of presarcopenia using body height and arm span for postmenopausal osteoporosis. Clin. Interv. Aging. 2020, 15, 357-361. [CrossRef] [PubMed]

46. Stanbury, R.M.; Graham, E.M. Systemic corticosteroid therapy side effects and their management. Br. J. Ophthalmol. 1998, 82, 704-708. [CrossRef]

47. Owczarek, J.; Jasińska, M.; Orszulak-Michalak, D. Drug-induced myopathies. An overview of the possible mechanisms. Pharmacol. Rep. 2005, 57, 23-34.

48. Kamen, G. Aging, resistance training, and motor unit discharge behavior. Can. J. Appl. Physiol. 2005, 30, 341-351. [CrossRef]

49. Jenkins, N.D.; Miramonti, A.A.; Hill, E.C.; Smith, C.M.; Cochrane-Snyman, K.C.; Housh, T.J.; Cramer, J.T. Greater neural adaptations following high- vs. low-load resistance training. Front. Physiol. 2017, 8, 331. [CrossRef]

50. Sipilä, S.; Törmäkangas, T.; Sillanpää, E.; Aukee, P.; Kujala, U.M.; Kovanen, V.; Laakkonen, E.K. Muscle and bone mass in middle-aged women: Role of menopausal status and physical activity. J. Cachexia Sarcopenia Muscle. 2020, 11, 698-709. [CrossRef]

51. García-Bustínduy, M.; Gantes, M.A. Corticoides y osteoporosis. Actas Dermo Sifiliogr. 2007, 98, 526-530. [CrossRef]

52. Greendale, G.A.; Sternfeld, B.; Huang, M.; Han, W.; Karvonen-Gutierrez, C.; Ruppert, K.; Cauley, J.A.; Finkelstein, J.S.; Jiang, S.-F.; Karlamangla, A.S. Changes in body composition and weight during the menopause transition. JCI Insight. 2019, 4, e124865. [CrossRef] [PubMed]

Publisher's Note: MDPI stays neutral with regard to jurisdictional claims in published maps and institutional affiliations.

(C) 2020 by the authors. Licensee MDPI, Basel, Switzerland. This article is an open access article distributed under the terms and conditions of the Creative Commons Attribution (CC BY) license (http://creativecommons.org/licenses/by/4.0/). 